\title{
Preventive Effects of Health-Food Drinks on the Obesity and DNA Damage of Mice Fed a High-Fat Diet under a Mild Stress
}

\author{
Minoru Higashimoto ${ }^{1,2,3,{ }^{*}}$, Chikako Toda $^{2}$, Nao Tokumoto ${ }^{2}$ and Arinobu Yamauchi ${ }^{2}$ \\ ${ }^{1}$ Awa Laboratory of Health-Food Drinks, Tokushima, Japan \\ ${ }^{2}$ Department of Food \& Nutrition, Suzugamine Women's College, Hiroshima, Japan \\ ${ }^{3}$ Graduate School of Human Life \& Science, Nagoya Keizai University, Aichi, Japan
}

\begin{abstract}
The body weight gain of mice fed an obesity-inducing diet is suppressed by a mild restraint stress, but at the same time, as previously reported, the stress induces DNA damage in the cells of multiple organs. In the present study, we attempted to prevent not only the obesity but also the DNA damage of mice fed an obesity-inducing diet under a mild restraint with commercially available health-food drinks such as fruit and vegetable juices, soymilks, vinegars and lactic acid bacteria drinks, which are rich in antioxidants. The body weight gain of young female mice fed a high-fat diet containing $20 \%$ fat for 4 weeks was considerably inhibited by restraint for 15 min per day in weeks 2 to 4 of 4 -week period. The inhibition was further promoted with the concomitant administration of health-food drinks noted above, and was accompanied by a decrease of periovular fat, a major abdominal fat in the female mice. The definite loss of energy intake in the mice given health-food drinks was approximately compensated by the energy of the drink administered. On the other hand, the increase of DNA damage generated by restraint in the cells of five organs - the liver, pancreas, spleen, heart and bone marrow - was markedly suppressed with the administration of these drinks. The results suggest that a combination of mild stress and intake of suitable health-food containing some antioxidants may inhibit lifestylerelated diseases including hyperlipidemia and obesity, which may contribute to the inhibition of metabolic syndrome and childhood obesity.
\end{abstract}

Keywords: health-food drinks, high-fat diet, restraint stress, obesity, DNA damage, dyslipidemia.

\section{INTRODUCTION}

It has been shown that mental and physiological stresses induce excess reactive oxygen species (ROS), worsen lifestyle-related diseases, cause cellular damage via oxidation of biomolecules, including DNA, proteins and polyunsaturated fatty acids, and finally induce a variety of diseases such as cancer, Parkinson's disease, and cardiovascular diseases [14]. In addition, unhealthy lifestyles such as excessive intake of high-fat diet and lack of exercise are prevalent among people today, and they can induce, promote and increase the lifestyle-related diseases [5-9]. The authors have been studying biological antioxidant defense systems induced by a variety of stresses and the antioxidant effects against reactive oxygen species [10-15], and are currently interested in the contribution of biological antioxidant defense system and food to the relationship between obesity and stress, investigating the possibility of utilizing beneficial effects of mild stress $[7,16]$. We previously reported that foodinduced dyslipidemia in mice could be accelerated by repeated fasting, but could be suppressed by repeated restraint, and that the DNA damage in cells of various organs was increased by repeated stress $[13,15]$. In these studies, we investigated the DNA damage in the

*Address correspondence to this author at the Awa Laboratory of Health-Food Drinks, 89 Kitabara, Awa-cho, Awa-shi, Tokushima 771-1703, Japan;

Tel: +81-90-2825-5049; E-mail: higashiyh@ybb.ne.jp cells of four murine organs susceptible to the influence of stress, using mild restraint stress. While some benefits of stress including suppression of body weight gain, improvement of fatty liver and activation of biological defense systems were observed, mild DNA damages were also detected in the cells of murine organs. Mild and beneficial stress is known to activate the brain and promote our health, especially in the elderly $[1,3]$. In the present study, we aimed to investigate whether a variety of health-food drinks commercially available may be able to prevent or reduce mild DNA damage in the cells with their antioxidative effects $[2,17,18]$, in combination with mild stress.

\section{EXPERIMENTAL METHODS}

\section{Animal Treatments}

Female weaning-age ICR mice (Japan SLC, Inc., Shizuoka, Japan) were divided into four to six groups as shown in Table 1. All of the experiments were performed in duplicate $(n=12)$. The mice assigned to the stress groups were fed high-fat diet (HFD) for 4 weeks and were subjected to fasting for 24 hours from 20:00 every other day, three times per week (F24), or to restraint with a tube for $15 \mathrm{~min}$ (R15) daily on weekdays, in weeks 2 to 4 of the 4-week study period as shown in the previous paper [13]. Control mice were fed standard diet (STD) (C1) or HFD (C2) for 4 weeks 
Table 1: Experimental Design

\begin{tabular}{|c|c|c|}
\hline Animal & \multicolumn{2}{|c|}{ Female ICR mice, 4 weeks of age, $n=6$} \\
\hline \multirow{2}{*}{$\begin{array}{l}\text { Health-food drink } \\
\text { group }\end{array}$} & \multicolumn{2}{|c|}{$\begin{array}{l}\text { 1. Fruit and vegetable juice }(12) \\
\text { Fv1-12 (40-65\% fruit juice }+35-60 \% \text { vegetable juice) }\end{array}$} \\
\hline & \multicolumn{2}{|c|}{$\begin{array}{l}\text { 2. Other Health-food drink (14) } \\
\text { Hd1-3 (soy milks),Hd4-7 (black vinegars), Hd8-10 (fruit juices), } \\
\text { Hd11-14 (lactic acid bacteria beverages) }\end{array}$} \\
\hline \multirow{4}{*}{ Control group } & C2. high-fat diet & High-fat diet control \\
\hline & F12. 12-h fasting & Obesity control \\
\hline & F24. 24-h fasting & Dyslipidemia control \\
\hline & 15-min restraint & Restraint control \\
\hline \multirow{2}{*}{ Treatment period } & Feeding period & 4 weeks \\
\hline & Restraint period & Weeks 2-4 \\
\hline
\end{tabular}

without being subjected to any stress. The HFD (Crea Japan, Osaka, Japan), specially prepared for our laboratory, was based on the AIN 76 diet [19], containing $9.6 \%$ corn oil, $13 \%$ beef tallow, $1 \%$ cholesterol, $0.2 \%$ cholic acid, and $433.0 \mathrm{kcal} / 100 \mathrm{~g}$. The STD (CE-2) containing $4.4 \%$ fat and 342.2 $\mathrm{kcal} / 100 \mathrm{~g}$ was purchased from Crea Japan. These solid-type diets were weighed and fed adequately. Total energy intakes were calculated as the sum of diet eaten by each treatment groups during the experiment. Cages of the animals were cleaned regularly during the fasting period to prevent coprophagy. Restraint was performed using a handmade restraint device, a $50-\mathrm{ml}$ plastic centrifuging tube modified for this experiment, as shown in the previous paper [15]. The device has twelve 3-mm diameter holes for sweating, one hole for tail-protecting, and four holes fitted with two steel rings for the animal's habitual teeth grinding and breathing. Volume of centrifuging tube was changed in accordance with the size of the animal. At the end of the treatment period, mice exposed to stress were fed HFD for approximately one day, and all the animals were fasted for 2 hours before they were sacrificed. All experimental procedures were approved by the Animal Care and Use Arrangements Meetings of Suzugamine Women's Coll. and of Nagoya Keizai Univ. and conformed to the guidelines established by the Japanese Ministry of Education, Culture, Sports, Science and Technology.

\section{Sample Preparation}

At the end of the 4-week study period, blood samples were collected from the supraorbital vein of the animals under pentobarbital anesthesia. Then, the liver, pancreas, spleen and heart were quickly removed from each animal, weighed and placed on ice. The bone marrow cells were collected from the femur and eluted with phosphate buffered saline.

\section{Comet Assay}

Aliquots of mouse organs (liver, pancreas, spleen and heart) were homogenized in glass-teflon homogenizers. The homogenates and bone marrow eluate were subjected to the Comet assay [20, 21] using the Comet Assay and Silver Staining Kits (Trevigen, Gaitherburg, MD, USA) and SYBR Gold (Invitrogen, Eugene, OR, USA). The comet images obtained by using an optical or a fluorescence microscope equipped with a digital camera were analyzed and were scored using a comet analysis software (CometAnalyzer, Youworks, Tokyo, Japan). The DNA damage was expressed as a ratio of the total comet intensity to the head intensity of DNA image from 100 cells of each sample.

\section{Statistical Analysis}

Data are presented as mean ( \pm SEM). Differences in means were analyzed using unpaired $t$-test with 
InStat 2.0 (GraphPad Software Inc.). Statistical significance was defined as $p<0.05$.

\section{RESULTS}

\section{Body Weight Change}

Body weights of control mice in the HFD control group (C2) and the 24-h fasted group (F24) were similarly increased and were higher than those of mice in the STD control group (C1), and those in the 12-h fasted group (F12) were highly increased as shown in Figure 1. On the other hand, the increase in body weight of the restraint group (R15) was clearly reduced by stress as shown in Figure 1. Body weight gains of mice in most of the twelve experimental drink groups (Fv1-12) were reduced to the level lower than those of the restraint group as shown in Figure 1. Similar tendencies were seen in the body weights of mice administered with the other fourteen health-food drinks as shown in Figure 2 (Hd1-14).

\section{Abdominal Fat Weight}

Weights of the periovular fat which is a major abdominal fat of female mice were increased in the
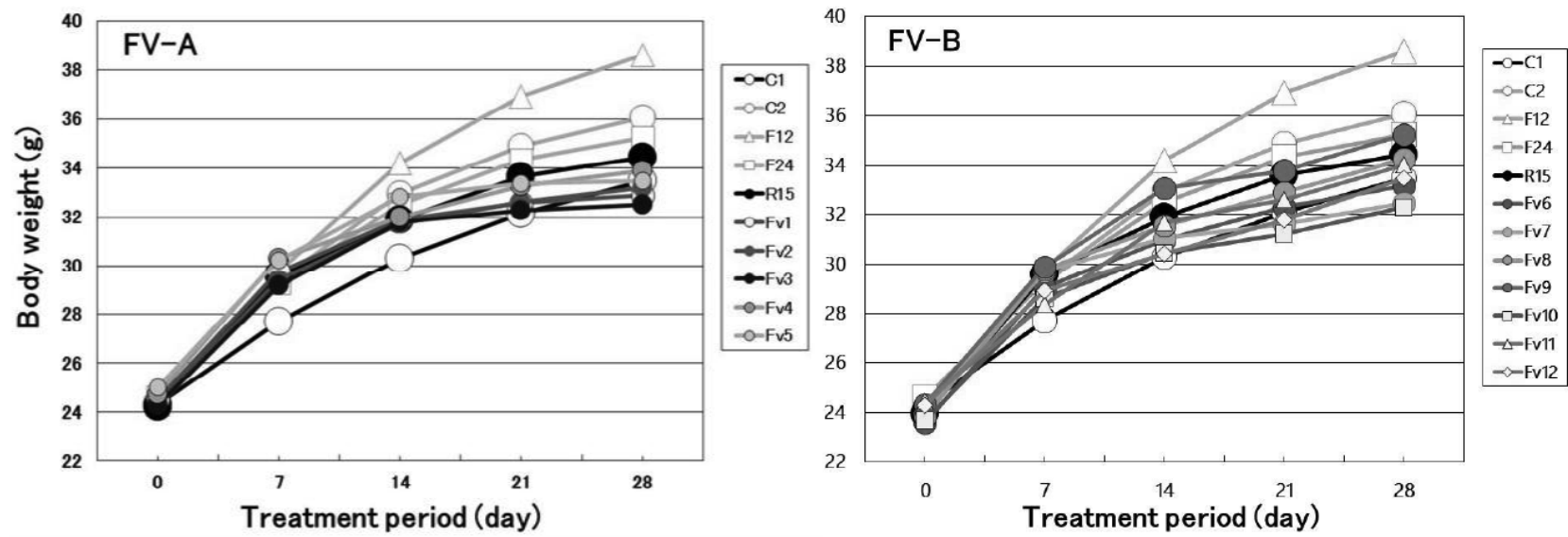

Figure 1: Change in body weight in mice of the control groups and the experimental drink groups exposed to restraint repeatedly for $15 \mathrm{~min}$ per day under concomitant administration of high-fat diet and one of twelve fruit and vegetable juices.

C1, standard diet control; C2, high-fat diet control; F12 and F24, high-fat diet plus fasting for 12 h and 24 h, respectively; R15, high-fat diet plus restraint for $15 \mathrm{~min}$. Mice were exposed to stress daily on weekdays in weeks 2 to 4 of the 4 -week study period, except for F24 (every other day) mice. Data of the control groups (C1, C2, F12, F24 and R15) and the experimental drink groups are presented as means obtained from two experimental groups for twelve fruit and vegetable juice groups (FV-A: Fv1-5 and FV-B: Fv6-12), $\mathrm{n}=12$.
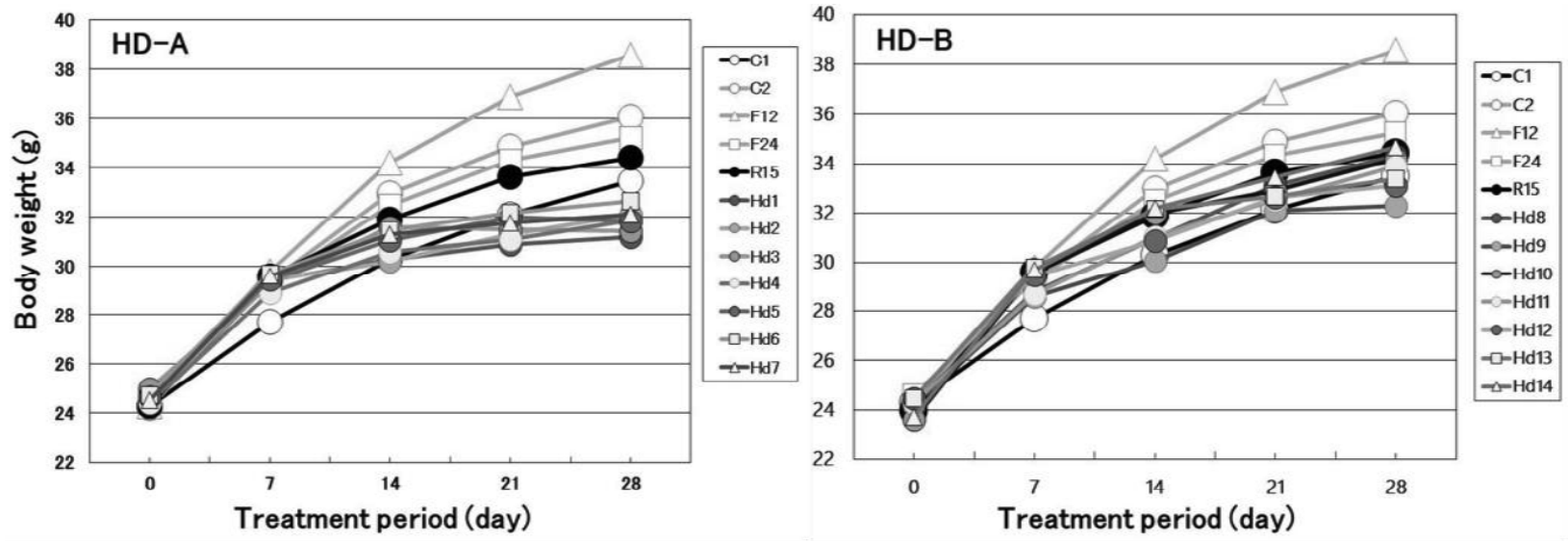

Figure 2: Change in body weight in mice of the control groups and the experimental drink groups exposed to restraint repeatedly for 15 min per day under concomitant administration of high-fat diet and one of the other fourteen health-food drinks.

C1, standard diet control; C2, high-fat diet control; F12 and F24, high-fat diet plus fasting for 12 h and 24 h, respectively; R15, high-fat diet plus restraint for $15 \mathrm{~min}$. Mice were exposed to stress daily on weekdays in weeks 2 to 4 of the 4 -week study period, except for F24 (every other day) mice. Data of the control groups (C1, C2, F12, F24 and R15) and the experimental drink groups are presented as means obtained for the other fourteen health-food drink groups (HD-A: Hd1-7 and HD-B: Hd814), $n=12$. 


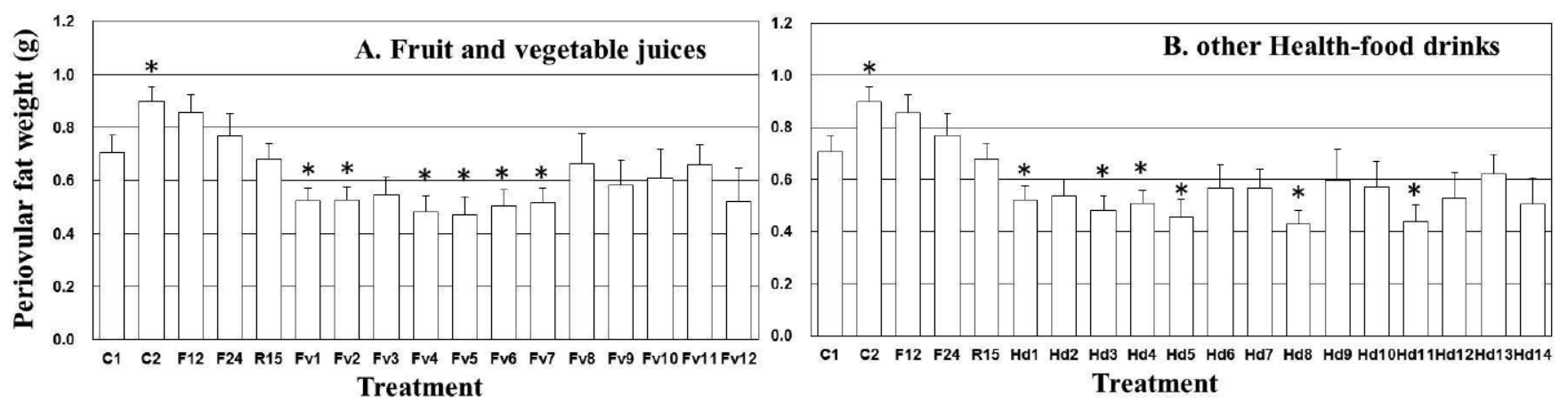

Figure 3: Periovular fat weight in mice of the control groups and the experimental drink groups exposed to restraint repeatedly for 15 min per day under concomitant administration of high-fat diet and one of twelve fruit and vegetable juices and the other fourteen health-food drinks.

C1, standard diet control; C2, high-fat diet control; F12 and F24, high-fat diet plus fasting for 12 h and 24 h, respectively; R15, high-fat diet plus restraint for $15 \mathrm{~min}$. Mice were exposed to stress daily on weekdays in weeks 2 to 4 of the 4 -week study period, except for F24 (every other day) mice. Data of the control groups (C1, C2, F12, F24 and R15) and the experimental drink groups are presented as mean \pm SEM obtained from twelve fruit and vegetable juice groups (A. Fv1-12) and from the other fourteen health-food drink groups (B. Hd1-14), $\mathrm{n}=12$. . * $p<0.05$ vs. corresponding R15 group.

HFD control group and the fat weights were gradually reduced in proportion to the intensity of the stress and the fat weights of mice in the restraint control group were reduced to the same level as those of the standard diet group as shown in Figure 3 . The fat weight was further reduced by the concomitant administration of most kinds of the twelve fruit and vegetable juices and the other health-food drinks similarly, as shown in Figure 3. There were significant differences between the weights for $50 \%$ of the fruit and vegetable juice groups and for $43 \%$ of the other health-food drink groups and that of the restraint control group.

The weights of other organs, that is, liver, pancreas, spleen and heart, did not show significant differences between the health-food drink groups and the restraint control group (data not shown).

\section{Total Energy Intake}

Total energy intake in each experiment group was expressed as the percentage to the total energy intake in the corresponding STD control group (C1) as shown in Figure 4. Relative energy intake of the HFD control group (C2) was higher than that for the corresponding STD control group. Both in the fruit and vegetable juice group and the other health-food drink group, total energy intakes of 12- and 24-h fasting (F12 and F24) and restraint (R15) groups were almost similar to that of the corresponding STD control group (C1). Although the energy intakes from diet in the fruit and vegetable

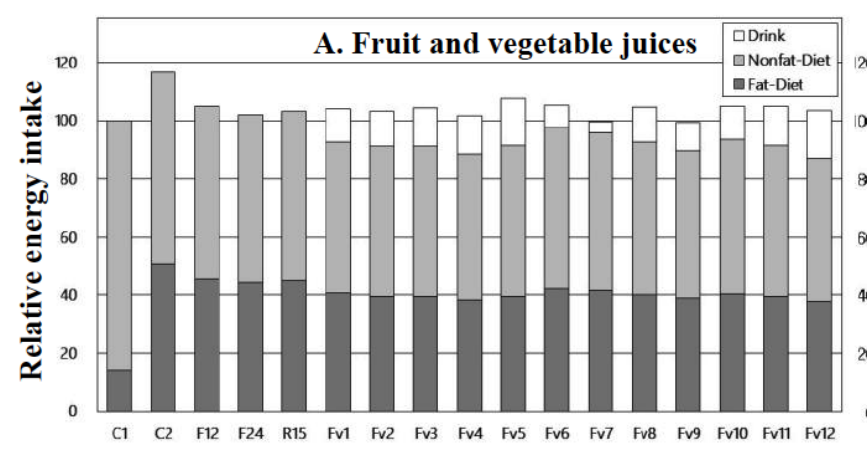

Treatment

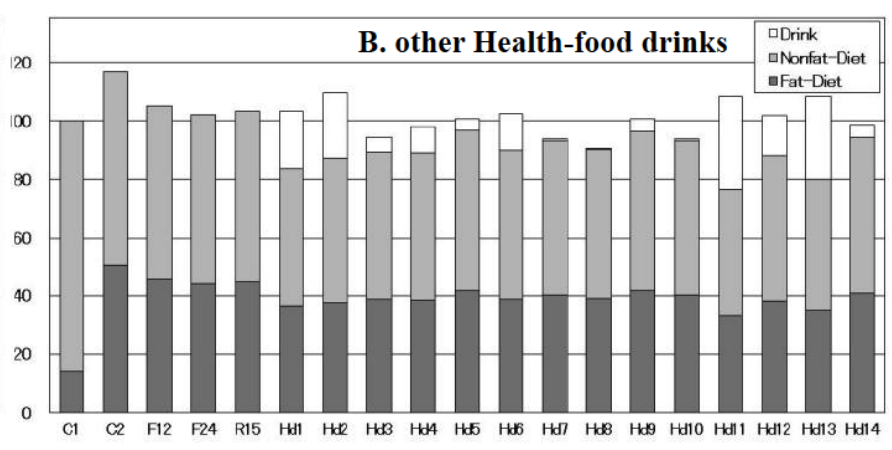

Treatment

Figure 4: Relative total energy intakes from fat- and non-fat-components in the diet and from drinks for mice of the control groups and the experimental drink groups exposed to restraint repeatedly for 15 min per day under concomitant administration of high-fat diet and one of twelve fruit and vegetable juices and the other fourteen health-food drinks.

C1, standard diet control; C2, high-fat diet control; F12 and F24, high-fat diet plus fasting for 12 h and 24 h, respectively; R15, high-fat diet plus restraint for $15 \mathrm{~min}$. Mice were exposed to stress daily on weekdays in weeks 2 to 4 of the 4 -week study period, except for F24 (every other day) mice. Data of the control groups (C1, C2, F12, F24 and R15) and the experimental drink groups are presented as mean obtained from two experimental groups for twelve fruit and vegetable juice groups (A. Fv112) and for the other fourteen health-food drink groups (B. Hd1-14), $n=2$. 

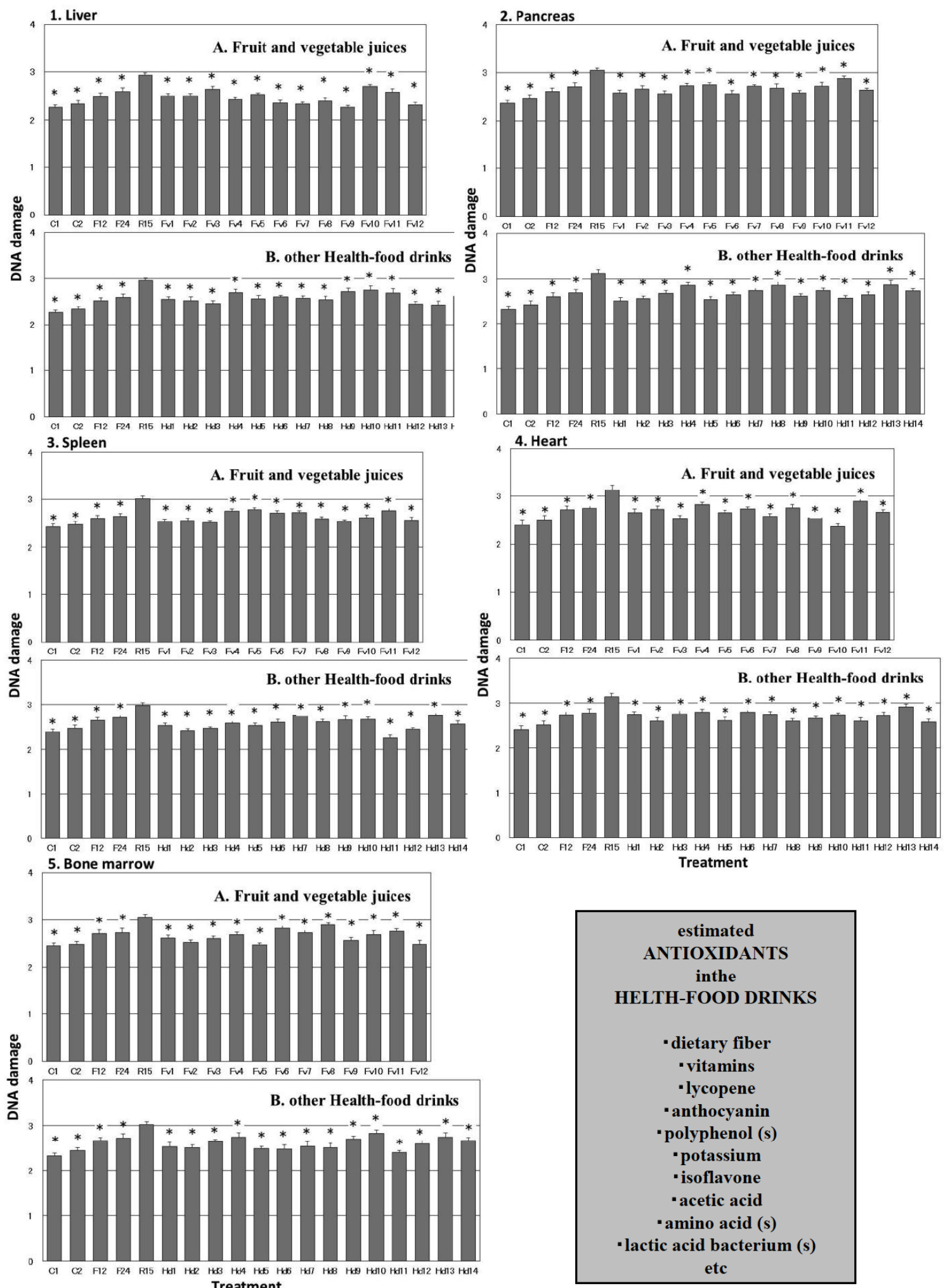

Figure 5: DNA damages in the (1) liver, (2) pancreas, (3) spleen, (4) heart and (5) bone marrow of the control groups, and the experimental drink groups exposed to restraint repeatedly for 15 min per day under concomitant administration of high-fat diet and one of twelve fruit and vegetable juices and the other fourteen health-food drinks.

C1, standard diet control; C2, high-fat diet control; F12 and F24, high-fat diet plus fasting for 12 h and 24 h, respectively; R15, high-fat diet plus restraint for $15 \mathrm{~min}$. Mice were exposed to stress daily on weekdays in weeks 2 to 4 of the 4 -week study period, except for F24 (every other day) mice. Data of the control groups (C1, C2, F12, F24 and R15) and the experimental drink groups for twelve fruit and vegetable juice groups (A. Fv1-12) and for the other fourteen health-food drink groups (B. Hd1-14) are presented as mean \pm SEM obtained from each 100 cells. * $p<0.05$ vs. corresponding R 15 group. 
groups were generally reduced a little compared with those of the corresponding restraint control groups (R15), the reduced energy was compensated by the energy from the fruit and vegetable juice. The total energy intake in some of the other health-food drink groups was a little lower than that of the restraint control group (R15) because of the low energy content of the health-food drinks, which may contain artificial sweetener(s). In general, the fat-energy intakes in all HFD groups were much higher than that in the STD control group (C1).

\section{DNA Damage}

DNA damage in the liver, pancreas, spleen, heart and bone marrow of control groups and experimental drink groups exposed to restraint repeatedly for $15 \mathrm{~min}$ per day under concomitant administration of high-fat diet and one of twelve fruit and vegetable juices or the other fourteen health-food drinks is shown in Figure $\mathbf{5}$. There were significant differences between the R15 group and all treatment groups.

\section{DISCUSSION}

Childhood obesity, which is associated with metabolic syndrome, diabetes, heart disease and cancer, is one of the most debilitating and serious public health issues worldwide [4, 8, 9]. Diabetes is among the most serious morbidities resulting from childhood obesity and its prevalence is now increasing globally. One in eleven adults in the world has diabetes according to the estimation by the International Diabetes Federation (IDF) [22]. Diabetes and its complications are major causes of death in most countries and the increase in prevalence of these diseases is seen not only in adults but also in child and adolescents. Diabetes is classified into type 1 and type 2 and approximately $87 \%$ to $91 \%$ of people with diabetes are estimated to have type 2, which is a typical lifestylerelated disease. Healthy eating habits and increased physical activity are highly recommended for preventing the risk of type 2 diabetes $[22,23]$.

In the present study, we documented that body weight gain in the mice was considerably reduced by repeated restraint for 15 minutes per day (Figures 1 and 2) and that the reduction of body weight gain was further promoted by concomitant administration of fruit and vegetable juices and other health-food drinks, with a decrease in abdominal fat weight (Figure 3). While some benefits of stress including suppression of body weight gain were observed, mild DNA damage was seen in the cells of murine organs as previously described [15], which was clearly suppressed by the concomitant administration of the health-food drinks (Figure 5). Both the decrease in body weight gain and the suppression of DNA damage may be due to the additive action and/or synergistic effects of the antioxidants including dietary fiber, vitamins, lycopene, anthocyanin, polyphenol, potassium, isoflavone, acetic acid, amino acid(s), and lactic acid bacteria, and so on $[2,17,18]$. It has been shown that mental and physiological stresses induce excess reactive oxygen species (ROS) and worsen lifestyle-related diseases [3, $5,6]$. The presence of excess ROS can cause cellular damage via oxidation of biomolecules, including DNA, proteins and polyunsaturated fatty acids, causing a variety of diseases such as cancer, Parkinson's disease, and cardiovascular disease [1, 2]. To reduce the formation of excess ROS is important for preventing the development of diseases.

Antioxidants are compounds abundant in fresh fruits and vegetables [2]. A number of publications have demonstrated that adequate consumption of plant foods is associated with decreased risk of chronic degenerative diseases, such as coronary heart disease, stroke, diabetes or certain types of cancer, whose risk-reducing effect are attributed to the components including phytochemicals, phytonutrients and several vitamins, minerals and fiber [24]. While many epidemiological studies have consistently shown that regular consumption of fruits and vegetables is strongly associated with reduced risk for developing chronic diseases, the actions of antioxidant nutrients alone do not explain the observed health effects of diet rich in fruits and vegetables, because, if taken alone, the individual antioxidants studied in clinical trials do not appear to have consistent preventive effects [25]. The development of synergistic effects of free radical scavengers in fruits and vegetables needs to be further investigated, and several pilot studies with vitamins such as ascorbic acid, tocopherol and $\beta$-carotene are currently underway $[18,26,27]$. We described here the suppression of DNA damage generated by mild stress in the cells of murine organs by the use of concomitant administration of health-food drinks mixed with fruits and vegetables commercially available.

Based on the results of this study, it was shown that DNA damage of the cells in multiple murine organs could be suppressed by the combination of health-food drinks and mild stress exposure, suggesting that some antioxidative foods may be able to prevent the development of lifestyle-related diseases associated with obesity and hyperlipidemia in combination with 
beneficial effects of mild stress $[7,16]$, not only in the experimental animals described here but also in humans.

\section{ACKNOWLEDGEMENTS}

Thanks are due to the laboratory staff of Suzugamine Women's College (Hiroshima, Japan) and Nagoya Keizai Univ. (Aichi, Japan) for their helpful support in the animal experiments, and to Dr. Professor Yoshinari Ohnishi (School of Medicine, The University of Tokushima, Tokushima, Japan) for the valuable support throughout the study.

\section{REFERENCES}

[1] Olinski R, Siomek A, Rozalski R, et al. Oxidative damage to DNA and antioxidant status in aging and age-related diseases. Acta Biochim Polonica 2007; 54: 11-26.

[2] Palafox-Carlos H, Ayala-Zavala JF, González-Aguilar GA. The role of dietary fiber in the bioaccessibility and bioavailability of fruit and vegetable antioxidants. J Food Sci 2011; 76: R6-R15.

http://dx.doi.org/10.1111/j.1750-3841.2010.01957.x

[3] Poljsak B. Strategies for reducing or preventing the generation of oxidative stress. Oxid Med Cell Longev 2011; 1-15. http://dx.doi.org/10.1155/2011/194586

[4] Marseglia L, Manti S, D'Angelo G, et al. Oxidative stress in obesity: a critical component in human diseases. Int $\mathrm{J} \mathrm{Mol}$ Sci 2015; 16: 378-400.

http://dx.doi.org/10.3390/ijms 16010378

[5] Fukuda S, Morimoto K. Lifestyle, stress and cortisol response: Review I, mental stress. Environ Health Prev Med 2001; 6: 9-14. http://dx.doi.org/10.1007/BF02897303

[6] Fukuda S, Morimoto K. Lifestyle, stress and cortisol response: Review II, lifestyle. Environ Health Prev Med 2001; 6: $15-21$.

http://dx.doi.org/10.1007/BF02897304

[7] Meydani M, Hasan ST. Dietary polyphenols and obesity. Nutrients 2010; 2: 737-51.

http://dx.doi.org/10.3390/nu2070737

[8] Güngör NK. Overweight and obesity in children and adolescents. J Clin Res Pediatr Endocrinol 2014; 6: 129-43. http://dx.doi.org/10.4274/jcrpe.1471

[9] Pulgaron ER, Delamater AM. Obesity and type 2 diabetes in children: epidemiology and treatment. Curr Diab Rep 2014; 14: 508.

http://dx.doi.org/10.1007/s11892-014-0508-y

[10] Kondoh M, Tsukada M, Kuronaga $M$, et al. Induction of hepatic metallothionein synthesis by endoplasmic reticulum stress in mice. Toxicol Lett 2004; 148: 133-9. http://dx.doi.org/10.1016/j.toxlet.2003.12.066

[11] Suzuki S, Tohma S, Futakawa N, Higashimoto M, Takiguchi M, Sato M. Induction of hepatic metallothionein by mitocho- ndrial oxidative stress in mice. J Health Sci 2005; 51: 533-7. http://dx.doi.org/10.1248/jhs.51.533

[12] Futakawa N, Kondoh M, Ueda S, et al. Involvement of oxidative stress in the synthesis of metallothionein induced by mitochondrial inhibitors. Biol Pharm Bull 2006; 29: 2016-20. http://dx.doi.org/10.1248/bpb.29.2016

[13] Higashimoto $M$, Isoyama $N$, Ishibashi $S$, et al. Tissuedependent preventive effect of metallothionein against DNA damage in dyslipidemic mice under repeated stresses of fasting or restraint. Life Sci 2009; 84: 569-75. http://dx.doi.org/10.1016/j.Ifs.2009.01.022

[14] Sato M, Ishibashi S, Higashimoto M, Kadota Y, Kawakami T, Suzuki S. Early changes induced by environmental stresses in insulin sensitivity-related genes. Eur J Pharmaco 2011; 1668: 472-6.

[15] Higashimoto $M$, Isoyama $N$, Ishibashi $S$, et al. Preventive effects of metallothionein against DNA and lipid metabolic damages in dyslipidemic mice under repeated mild stress. J Med Invest 2013; 60: 240-8.

http://dx.doi.org/10.2152/jmi.60.240

[16] Kouda K, Iki M. Beneficial effects of mild stress (hormetic effects): Dietary restriction and health. J Physiol Anthropol 2010; 29: 127-32.

http://dx.doi.org/10.2114/jpa2.29.127

[17] Machlin LJ, Bendich A. Free radical tissue damage: protective role of antioxidant nutrients. FASEB J 1987; 1: 441-5.

[18] Jacob RA, Burri BJ. Oxidative damage and defense. Am J Clin Nutr 1996; 63: 985S-90.

[19] Bieri JG, Stoewsand GS, Briggs GM, Phillips RW, Woodard JC, Knapka JJ. Report of the American Institute of Nutrition Ad Hoc Committee on standards for nutritional studies. J Nutr 1977; 107: 1340-8.

[20] Sasaki YF, Tsuda S, Izumiyama F, Nishidate E. Detection of chemically induced DNA lesions in multiple mouse organs (liver, lung, spleen, kidney, and bone marrow) using the alkaline single cell gel electrophoresis (Comet) assay. Mutat Res 1997; 388: 33-44.

http://dx.doi.org/10.1016/S1383-5718(96)00133-7

[21] Collins AR. The comet assay for DNA damage and repair Mol Biotech 2004; 26: 249-61.

http://dx.doi.org/10.1385/MB:26:3:249

[22] IDF Diabetes Atlas $7^{\text {th }}$ ed., 2015.

[23] Katz DL. Diet and diabetes: lines and dots. J Nutr 2014; 144 567s-70. http://dx.doi.org/10.3945/jn.113.182923

[24] Lamprecht M, Obermayer G, Seebauer W. Influence of mixed fruit and vegetable concentrates on redox homeostasis and immune system of exercising people. Antioxidants in Sport Nutrition. Chapter 12: Florida; Boca Raton: CRC Press/Taylor \& Francis 2015.

[25] Rui Hai Liu. Potential synergy of phytochemicals in cancer prevention: mechanism of action. J Nutr 2004; 134: 3479S-85.

[26] Diplock AT, Charleux JL, Crozier-Villi G, et al. Functional food science and defence against reactive oxidative species. Br J Nutr 1998; 80(Suppl 1): S77-112. http://dx.doi.org/10.1079/BJN19980106

[27] Juan CA, Veronica F-S, Pedro M-R, Maria LV, et al. Frontiers in aging. Neurosc 2015; 7: 1-7.

http://dx.doi.org/10.6000/1929-4247.2016.05.03.3

(c) 2016 Higashimoto et al.; Licensee Lifescience Global.

This is an open access article licensed under the terms of the Creative Commons Attribution Non-Commercial License (http://creativecommons.org/licenses/by-nc/3.0/) which permits unrestricted, non-commercial use, distribution and reproduction in any medium, provided the work is properly cited. 